\title{
Taxas de serviço, concepção e prenhez de vacas nelore tratadas com gonadotrofina coriônica humana e $17 \beta$-estradiol após a inseminação artificial em tempo fixo
}

Rui MACHADO'

Marco Aurélio Carneiro Meira BERGAMASCHI ${ }^{1}$ Rogério Taveira BARBOSA ${ }^{1}$ Ed Hoffmann MADUREIRA ${ }^{2}$ Maurício Melo de ALENCAR $^{1}$

Mário BINELLI ${ }^{2}$

\section{Correspondência para:}

Dr. Rui Machado, Embrapa Pecuária Sudeste-Fazenda Canchim, Caixa Postal 339 - 13.560 -970, São Carlos, SP. rui@cppse.embrapa.br

Recebido para publicação: 29/06/2005 Aprovado para publicação: 24/04/2008

\author{
1 - Embrapa Pecuária Sudeste, São Carlos-SP \\ 2 - Departamento de Reprodução Animal da Faculdade de Medicina \\ Veterinária da Universidade de São Paulo, Pirassununga-SP
}

\section{Resumo}

Avaliaram-se estratégias para reduzir a mortalidade embrionária em bovinos. Vacas Nelore (Bos taurus indicus) foram inseminadas em tempo fixo (IATF - $\mathrm{D}_{0}$ ) após o protocolo GnRH/PGF ${ }_{2 \mathrm{a}} / \mathrm{GnRH}$ e divididas nos grupos: Controle $(\mathrm{Gc} ; \mathrm{n}=55), \mathrm{hCG}\left(\mathrm{G}_{\mathrm{hCG}} ; \mathrm{n}=55\right)$ recebeu $3000 \mathrm{UI}$ da hCG no $\mathrm{D}_{5}, \mathrm{E}_{2}\left(\mathrm{G}_{\mathrm{E} 2} ; \mathrm{n}=55\right)$ recebeu $5 \mathrm{mg}$ de $17_{\mathrm{b}}$ Estradiol $\left(\mathrm{E}_{2}\right)$ no $\mathrm{D}_{12}$ e hCG $/ \mathrm{E}_{2}\left(\mathrm{G}_{\mathrm{hCG} / \mathrm{E} 2} ; \mathrm{n}=55\right)$ recebeu $3000 \mathrm{UI}$ da hCG no $\mathrm{D}_{5}$ e $5 \mathrm{mg}$ do $\mathrm{E}_{2}$ no $\mathrm{D}_{12}$. A IA $\mathrm{TF}_{\mathrm{TF}}$ foi o $1^{\circ}$ dia da estação de reprodução de 64 dias. Usou-se o teste do $\mathrm{c}^{2}$ para comparar as taxas de serviço (TS), concepção (TC) e prenhez à IA TF $_{\text {(TP }}$ IATF $)$, às IAs de repasse e acumuladas. A TS e a TC das IAs de repasse não diferiu $(\mathrm{P}>0,10)$. Houve diferenças nas $\mathrm{TP}_{\text {IATF }}(\mathrm{P}<0,05)$, pois a $\mathrm{TP}_{\text {IATF }}$ do $\mathrm{G}_{\mathrm{E} 2}(5,4 \%)$ foi a mais baixa e a do $\mathrm{Gc}(34,5 \%)$ e $\mathrm{G}_{\mathrm{hCG}}(36,5 \%)$ tenderam $(\mathrm{P}<0,10)$ ser maiores que a do $\mathrm{G}_{\mathrm{hCG} / \mathrm{E} 2}(18,2 \%)$. Não houve efeito da hCG sobre as taxas de prenhez. $\mathrm{O} \mathrm{E}_{2}$ reduziu a TP $\mathrm{TP}_{\text {IATF }}$ e ao final da estação as vacas que receberam o $\mathrm{E}_{2}\left(\mathrm{G}_{\mathrm{E} 2}+\mathrm{G}_{\mathrm{hCG} / \mathrm{E} 2}\right)$ tiveram TP $(66,4 \%)$ mais baixa $(\mathrm{P}<0,05)$ de que aquelas $(80,9 \%)$ que não receberam $\left(G_{C}+G_{h C G}\right)$. Em conclusão, as estratégias adotadas de manipulação luteínica ou folicular não aumentaram as taxas de prenhez em vacas Nelore após uma IA ${ }_{\mathrm{TF}}$.

\section{Introdução}

A situação da economia mundial exige alta rentabilidade do agronegócio. ${ }^{1} \mathrm{No}$ caso da pecuária de corte nacional, a taxa de desfrute situada próxima aos $21 \%{ }^{2}$ está muito aquém do potencial real para a atividade. A baixa fertilidade dos rebanhos brasileiros é um dos fatores desse desempenho insatisfatório. Dentre as causas de fertilidade baixa em bovinos de corte destacam-se perdas embrionárias de até $40 \%$, as quais ocorrem no "período crítico" de reconhecimento da prenhez (entre 15 e 19 dias após a fertilização ${ }^{3}$ ). Nesse período, o mecanismo da luteólise deve ser bloqueado por intermédio de sinais bioquímicos enviados pelo concepto para a unidade materna, a qual por sua vez deve prover condições uterinas adequadas para o desenvolvimento otimizado do concepto.

Diversos estudos revisados por Binelli et al. ${ }^{4}$ implicaram o estradiol nos fenômenos que desencadeiam a luteólise. Portanto, o sucesso da manutenção da prenhez depende da inibição da produção e/ou ação daquele esteróide. Neste caso, uma estratégia para minimizar o estrógeno circulante é remover o folículo ovariano dominante (FD) que está presente durante o período crítico $^{4}$, ocasião de alta atividade esteroidogênica pelo $\mathrm{FD}^{5}$. Uma abordagem farmacológica da remoção é a aplicação de estrógenos exógenos, que por mecanismo de retroalimentação negativa inibem o crescimento do $\mathrm{FD}^{4}$. De fato, a aplicação do $17_{\mathrm{b}}$-Estradiol exógeno no $12^{\circ}$ dia do ciclo estral reduziu o número de dias dentro do período crítico $\left(\mathrm{D}_{15}\right.$ ao $\left.\mathrm{D}_{19}\right)$ nos 
quais havia um $\mathrm{FD}^{6}$.

A progesterona materna circulante prepara o útero para assegurar o desenvolvimento do concepto. ${ }^{7,8}$ De fato, concentrações plasmáticas baixas de progesterona na fase luteínica relacionam-se a embriões menos desenvolvidos ${ }^{8}$ e taxas de concepção menores. ${ }^{7}$ Por outro lado, a suplementação com progesterona aumenta a taxa de crescimento embrionário e também a capacidade em produzir a molécula sinalizadora da sua presença no útero (interferon-t). ${ }^{9}$

Uma estratégia para aumentar a progesterona plasmática consiste na aplicação de hCG entre os dias 4 e 7 do ciclo estral para induzir a formação de um corpo lúteo acessório. ${ }^{10,11,12}$ Com efeito, numa revisão de 17 estudos individuais verificou-se que, vacas suplementadas com progesterona após a inseminação artificial obtiveram taxas de concepção aproximadamente $5 \%$ mais altas do que vacas não suplementadas. ${ }^{7}$

Neste experimento visou-se comparar a eficácia de diferentes estratégias para redução da mortalidade embrionária por meio da manipulação luteínica e folicular após uma inseminação artificial (IA) em tempo fixo $\left(\mathrm{IA}_{\mathrm{TF}}\right)$. As abordagens foram: 1) otimização da função luteínica pela administração da hCG no $5^{\circ}$ dia após a IA $\mathrm{T}_{\mathrm{TF}}$, 2 ) reprogramação da função folicular pela remoção farmacológica do folículo dominante ao final da fase luteínica por meio da aplicação do 17 -estradiol no $12^{\circ}$ dia após a IA $_{\mathrm{TF}}$, e $3^{\circ}$ ) combinação das duas abordagens anteriores. Para tanto, foram comparadas entre os grupos as taxas de prenhez à $\mathrm{IA}_{\mathrm{TF}}$ e às inseminações feitas nos ciclos naturais subsequentes dentro de uma estação reprodutiva de 64 dias.

\section{Material e Método}

O experimento foi desenvolvido na Fazenda Canchim da Embrapa Pecuária Sudeste, localizada em São Carlos, região central do estado de São Paulo (22 $02^{\circ}$ ' S e $47^{\circ} 54^{\prime} \mathrm{W}$ ). O clima é tropical de altitude, caracterizado como verão quente e inverno seco. Entre 1970 e 1997, a média da temperatura anual foi de $21,3^{\circ} \mathrm{C}$ e a precipitação pluvial média anual foi de 1353 milímetros (mm). Foram utilizadas 220 vacas adultas da raça Nelore (Bos taurus indicus), com mais de 240 dias pós-parto e sem bezerro ao pé. No início do experimento, todas fêmeas foram pesadas e avaliadas quanto ao escore de condição corporal (ECC; escala de 1 a 9). Foi realizado exame ginecológico por palpação e ultra-sonografia transretal. Todas vacas tinham 5ECC $£ 7$ e possuíam ovários de comprimento entre 15 e $30 \mathrm{~mm}$ com corpo lúteo ou com crescimento folicular associado à turgidez uterina. $\mathrm{O}$ manejo foi intensivo em sistema de rotação de pastagem com capins Brachiaria decumbens, Brachiaria brizantha var. Marandu, Panicum maximum var. Tanzânia e livre acesso a sal mineralizado e água.

As vacas tiveram o ciclo estral sincronizado pelo método OvSynch ${ }^{13}$, que consistiu numa injeção intramuscular (IM) de $8 \mathrm{mg}$ do acetato de buserelina (Acetato de buserelina $a^{\hat{a}}$ Centro Paulista de Desenvolvimento Farmacotécnico), seguida por $0,150 \mathrm{mg}$ de d-cloprostenol (Prolise ${ }^{\hat{a}}$, Tecnopec) - IM 7 dias mais tarde e outra aplicação IM de GnRH ( $\left.\mathrm{D}_{\text {zero }}\right) 48$ horas após esta última. A IA $\mathrm{ITF}_{\mathrm{TF}}$ ocorreu 16 horas depois da $2^{a}$ aplicação do GnRH usando sêmen de touros distintos distribuídos por sorteio aleatório entre os grupos. A IA ${ }_{\mathrm{TF}}$ foi considerada como o $1^{\circ}$ dia de uma estação reprodutiva de 64 dias. As fêmeas foram estratificadas por ordem de parto, peso vivo e ECC para o sorteio aleatório em quatro grupos com 55 vacas cada. O delineamento experimental foi o de blocos casualizados generalizados, com os grupos: Controle (Gc) - não recebeu tratamento adicional; hCG $\left(G_{\text {hCG }}\right)$ - recebeu 3000 UI da hCG (Vetecor $5000^{2}$, Laboratórios Calier S.A) - IM no $\mathrm{D}_{5}$; 17b-estradiol $\left(\mathrm{G}_{\mathrm{E} 2}\right)$ recebeu $5 \mathrm{mg}$ de $17 \mathrm{~b}$ estradiol (17beta-Estradiol ${ }^{\hat{a}}$, Centro Paulista de Desenvolvimento Farmacotécnico) - IM no $\mathrm{D}_{12}$; e hCG/17b-estradiol $\left(\mathrm{G}_{\mathrm{hCG} / \mathrm{E} 2}\right)$ recebeu $3000 \mathrm{UI}$ da hCG no $\mathrm{D}_{5}$ e $5 \mathrm{mg}$ do 17b-estradiol no $\mathrm{D}_{12}$. Foi procedida a 
observação do estro, por 30 minutos, duas vezes ao dia (07:00 horas e às 16:30 horas) do $\mathrm{D}_{5}$ até $\mathrm{D}_{64}$. Incluíram-se dois rufiões por grupo, equipados com marcador "chinball" e mantidos até o final da estação reprodutiva.

O diagnóstico de prenhez foi realizado por ultra-sonografia no $D_{31}$ e 35 dias após o final da estação reprodutiva $\left(\mathrm{D}_{99}\right)$ com um aparelho Aloka, modelo SSD 500, em modo-B com transdutor de 7,5 MHz, por via retal e segundo procedimento padronizado para bovinos.

As taxas de prenhez (razão entre o número de vacas prenhes e o total de vacas disponíveis àquela IA); de serviço (razão entre o número de vacas inseminadas e o número total de vacas disponíveis em cada inseminação; e de concepção (razão entre o número de vacas prenhes à ultra-sonografia e o de vacas inseminadas em cada IA) foram mensuradas em resposta à $\mathrm{IA}_{\mathrm{TF}}$, à $1^{\mathrm{a}} \mathrm{IA}$ de repasse e à $2^{a}$ IA de repasse, bem como determinados seus valores cumulativos à cada IA de repasse e ao final da estação de reprodução. As taxas foram tomadas como proporções e analisadas pelo teste do Quiquadrado $\left(c^{2}\right)$ utilizando o Proc FREQ do programa SAS System for Windows ${ }^{14}$. Os efeitos dos grupos foram aceitos para a significância de até $5 \%$ de probabilidade $(\mathrm{P}<0,05)$ e conceituou-se como tendência quando os efeitos estiveram situados entre as probabilidades de 5 e $10 \%$ $(0,05<\mathrm{P}<0,10)$. Foram ainda comparadas as taxas "dentro" de cada grupo relativas às ocasiões de inseminação $\left(\mathrm{IA}_{\mathrm{TF}}, 1^{\mathrm{a}} \mathrm{IA}\right.$ de repasse e $2^{a}$ IA de repasse).

\section{Resultados}

Houve diferenças $(P<0,05)$ nas taxas de prenhez à $\mathrm{IA}_{\mathrm{TF}}$ entre grupos (Tabela 1). A taxa de prenhez do $G_{C}$ tendeu $\left(\mathrm{c}^{2}=3,79_{\mathrm{GL}=1} ; 0,10>\mathrm{P}>0,05\right)$ ser maior $(34,5 \%)$ que a do grupo hCG/ $\mathrm{E}_{2}(18,2 \%)$ e foi superior $(P<0,05)$ ao $G_{E 2}$. Não houve diferença $(\mathrm{P}>0,10)$ entre os grupos para a TP à $1^{2}$ IA de repasse $\left(\chi_{\mathrm{gl}=3}^{2}=4,14\right)$ ou para a $2^{\mathrm{a}}$ Ia de repasse $\left(\chi_{\mathrm{gl}=3}^{2} \stackrel{\mathrm{gl}}{=} 1,12\right)$. Porém, a taxa de prenhez acumulada até a $1^{a}$ IA de repasse foi menor $(P<0,05)$ para o $G_{\mathrm{hCG} / \mathrm{E} 2}$. A sincronização inicial da ovulação concentrou a manifestação de estro e a realização de inseminações dentro da estação de reprodução entre os dias 15 e 25 e entre os dias 35 e 48 (Figura 1). Houve tendências $(0,10>\mathrm{P}>0,05)$ das taxas de prenhez acumuladas (Tabela 1) diferirem entre os grupos controle e $\mathrm{E}_{2}\left(\chi^{2}=3,06_{\mathrm{GL}=1} ; 81,8 \%\right.$ e $67,3 \%$, respectivamente), controle e hCG/ $\mathrm{E}_{2} \quad\left(\chi^{2}=3,79_{\mathrm{GL}=1} ; 81,8 \%\right.$ e $65,4 \%$, respectivamente), bem como entre os grupos hCG e hCG $/ \mathrm{E}_{2}\left(\chi^{2}=2,93_{\mathrm{GL}=1} ; 80,0 \%\right.$ e $65,4 \%$, respectivamente).

Outra análise foi realizada considerando grupos de vacas que receberam ou não a hCG e que receberam ou não o 17_-Estradiol (Tabela 2). O efeito da hCG sobre as taxas de prenhez à $\mathrm{IA}_{\mathrm{TF}} \mathrm{e}$ acumulada não foi significativo. A taxa de prenhez acumulada das vacas que receberam o $17_{b}$-estradiol $\left(G_{\mathrm{E} 2}+G_{\mathrm{hCG} / \mathrm{E} 2}\right)$ foi menor $(66,4 \% ; \mathrm{P}<0,05)$ que das vacas que não o receberam $(80,9 \%)$.

Treze das 220 vacas usadas não se tornaram prenhes e nem retornaram ao estro depois da $\mathrm{IA}_{\mathrm{TT}}$, portanto a taxa de ciclicidade ao longo da estação de reprodução não alcançou $100 \%$. As taxas de serviço para a $1^{a}$ e $2^{a}$ IA de repasse não diferiram $(\mathrm{P}>0,05)$ entre os grupos (Tabela 1) e não houve diferença $(\mathrm{P}>0,05)$ entre as taxas de serviço observadas à $1^{2}$ ou à $2^{2}$ IA de repasse "dentro" de grupo (Tabela 1). No entanto, a taxa de serviço à $\mathrm{IA}_{\mathrm{TF}}(100,0 \%)$ foi maior $(\mathrm{P}<0,05)$ do que as taxas de serviço em qualquer das duas inseminações de repasse (comparação dentro de grupo) para qualquer dos grupos (Tabela 1). Ainda com relação à $1^{2}$ IA de repasse, houve nove inseminações antes do $\mathrm{D}_{18}$. Portanto, a incidência de inseminações realizadas em ciclos curtos após o OvSynch foi de 10,7\% (9/84).

As taxas de concepção para a $1^{2}$ e $2^{2}$ IA de repasse não diferiram $(\mathrm{P}>0,05)$ entre os grupos (Tabela 1) e não houve diferença $(\mathrm{P}>0,05)$ entre as taxas de concepção observadas à $1^{\text {a }}$ ou à $2^{2}$ IA de repasse "dentro" de grupo (Tabela 1). No entanto, 
Tabela 1 - Taxas de serviço (TS), concepção (TC) e prenhez (TP) 1 à inseminação artificial (IA) em tempo fixo ( ${ }_{\text {IATE }}$ ),

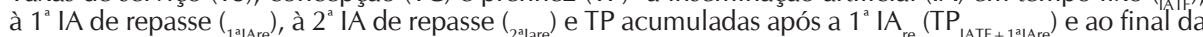
estação $\left(\right.$ TP $\left._{\text {acum }}\right)$. São Carlos, 2004

\begin{tabular}{|c|c|c|c|c|c|}
\hline \multirow{2}{*}{ Taxas } & \multicolumn{4}{|c|}{ Grupo } & \multirow{2}{*}{$\chi^{2} \mathrm{GL}=3$} \\
\hline & Controle & $\mathrm{hCG}$ & $\mathrm{E}_{2}$ & $\mathrm{hCG} / \mathrm{E}_{2}$ & \\
\hline \multicolumn{6}{|l|}{ Prenhez } \\
\hline$\overline{\mathrm{TP}_{\mathrm{IATF}}}$ & $\begin{array}{c}34,5 \% \%^{\mathrm{ab}} \\
(19 / 55)\end{array}$ & $\begin{array}{l}36,4 \% 0^{a} \\
(20 / 55)\end{array}$ & $\begin{array}{l}5,4 \%^{c} \\
(3 / 55)\end{array}$ & $\begin{array}{l}18,2 \% \mathrm{~b} \\
(10 / 55)\end{array}$ & $22,40 * *$ \\
\hline $\mathrm{TP}_{1^{2} \mathrm{IAre}}$ & $\begin{array}{c}55,5 \% \\
(20 / 36)\end{array}$ & $\begin{array}{c}42,8 \% \\
(15 / 35)\end{array}$ & $\begin{array}{c}40,4 \% \\
(21 / 52)\end{array}$ & $\begin{array}{c}33,3 \% \\
(15 / 45)\end{array}$ & $4,14^{\mathrm{ns}}$ \\
\hline $\mathrm{TP}_{2^{\mathrm{a}} \mathrm{IAre}}$ & $\begin{array}{l}37,5 \% \\
(6 / 16)\end{array}$ & $\begin{array}{c}45 \% \\
(9 / 20)\end{array}$ & $\begin{array}{c}38,7 \% \\
(12 / 31)\end{array}$ & $\begin{array}{l}30,0 \% \\
(9 / 30)\end{array}$ & $1,12^{\mathrm{ns}}$ \\
\hline \multicolumn{6}{|l|}{ Serviço } \\
\hline $\mathrm{TS}_{\mathrm{IATF}}$ & $100,0 \%{ }^{\mathrm{A}}$ & $100,0 \% \mathrm{~A}$ & $100,0 \% \mathrm{~A}$ & $100,0 \% \mathrm{~A}$ & ns \\
\hline $\mathrm{TS}_{1^{2} \mathrm{IAre}}$ & $\begin{array}{l}72,2 \%^{\mathrm{B}} \\
(26 / 36)\end{array}$ & $\begin{array}{l}65,7 \% \%^{\mathrm{B}} \\
(23 / 35)\end{array}$ & $\begin{array}{l}55,7 \%^{\mathrm{B}} \\
(29 / 52)\end{array}$ & $\begin{array}{l}57,8 \%{ }^{\mathrm{B}} \\
(26 / 45)\end{array}$ & $2,50^{\mathrm{ns}}$ \\
\hline $\mathrm{TS}_{2^{\mathrm{a}} \mathrm{IAre}}$ & $\begin{array}{c}50,0 \%{ }^{\mathrm{B}} \\
(8 / 16)\end{array}$ & $\begin{array}{l}65,0 \% \text { B } \\
(13 / 20)\end{array}$ & $\begin{array}{l}74,2 \%{ }^{\mathrm{B}} \\
(23 / 31)\end{array}$ & $\begin{array}{l}63,3 \%{ }^{\mathrm{B}} \\
(19 / 30)\end{array}$ & $2,59^{\mathrm{ns}}$ \\
\hline \multicolumn{6}{|l|}{ Concepção } \\
\hline$\overline{\mathrm{TC}_{\mathrm{IATF}}}$ & $34,5 \%$ ab, B & $36,4 \% \%^{a, B}$ & $5,4 \% c, B$ & $18,2 \%$ b, B & $22,40 * *$ \\
\hline $\mathrm{TC}_{1^{\mathrm{a}} \mathrm{IAre}}$ & $\begin{array}{l}76,9 \%{ }^{A} \\
(20 / 26)\end{array}$ & $\begin{array}{l}65,2 \% A \\
(15 / 23)\end{array}$ & $\begin{array}{l}72,4 \%{ }^{A} \\
(21 / 29)\end{array}$ & $\begin{array}{l}57,7 \% \text { A } \\
(15 / 26)\end{array}$ & $2,28^{\text {ns }}$ \\
\hline $\mathrm{TC}_{2^{\mathrm{a}}}$ IAre & $\begin{array}{c}75,0 \% \mathrm{~A} \\
(6 / 8)\end{array}$ & $\begin{array}{c}69,2 \% \mathrm{~A} \\
(9 / 13)\end{array}$ & $\begin{array}{l}52,2 \% \mathrm{~A} \\
(12 / 23)\end{array}$ & $\begin{array}{c}47,4 \% \mathrm{~A} \\
(9 / 19)\end{array}$ & $2,72^{\mathrm{ns}}$ \\
\hline \multicolumn{6}{|c|}{ Prenhez acumulada } \\
\hline $\mathrm{TP}_{\text {IATF }+1^{2} \mathrm{IAre}}$ & $\begin{array}{l}70,1 \% \%^{a} \\
(39 / 55)\end{array}$ & $\begin{array}{l}63,3 \% \%^{a} \\
(35 / 55)\end{array}$ & $\begin{array}{c}43,6 \% \mathrm{ab} \\
(25 / 55)\end{array}$ & $\begin{array}{l}38,2 \% \mathrm{~b} \\
(21 / 55)\end{array}$ & $10,90 * *$ \\
\hline $\mathrm{TP}_{\mathrm{acam}}$ & $\begin{array}{r}81,8 \% \\
(45 / 55)\end{array}$ & $\begin{array}{c}80,0 \% \\
(44 / 55)\end{array}$ & $\begin{array}{c}67,3 \% \\
(37 / 55)\end{array}$ & $\begin{array}{r}65,4 \% \\
(36 / 55)\end{array}$ & $6,01 *$ \\
\hline
\end{tabular}

1. * $=0,10>\mathrm{P}>0,05 ; * *=\mathrm{P}<0,05 ; \mathrm{ns}=$ não-significativo $(\mathrm{P} \geq 0,10)$; letras minúsculas superscritas distintas na mesma linha indicam diferença entre grupos $\left(\mathrm{P}<0,05 ; \mathrm{GL} \chi^{2}=1\right)$; letras maiúsculas superscritas distintas na mesma coluna indicam diferença dentro de grupo para aquela taxa comparada $\left(\mathrm{P}<0,05 ; \mathrm{GL} \chi^{2}=1\right)$.

as taxas de concepção à $\mathrm{IA}_{\mathrm{TF}}$ foram menores $(\mathrm{P}<0,05)$ do que as taxas de concepção em qualquer das duas inseminações de repasse (dentro de grupo) para qualquer dos grupos (Tabela 1).

\section{Discussão}

As estratégias adotadas não resultaram em aumento nas taxas de prenhez à $\mathrm{IA}_{\mathrm{TF}}$. A expectativa de se obter uma taxa de prenhez maior pela otimização da função luteínica em vacas suplementadas com hCG não se confirmou. Do mesmo modo, o $17_{\mathrm{b}}$ estradiol não bloqueou nem retardou a luteólise e em verdade reduziu as taxas de prenhez à $\mathrm{IA}_{\mathrm{TF}}$.

A hCG consistentemente otimiza a função luteínica pela indução da formação de corpo lúteo acessório e pelo aumento da 


\section{Curvas de Sobreviência}

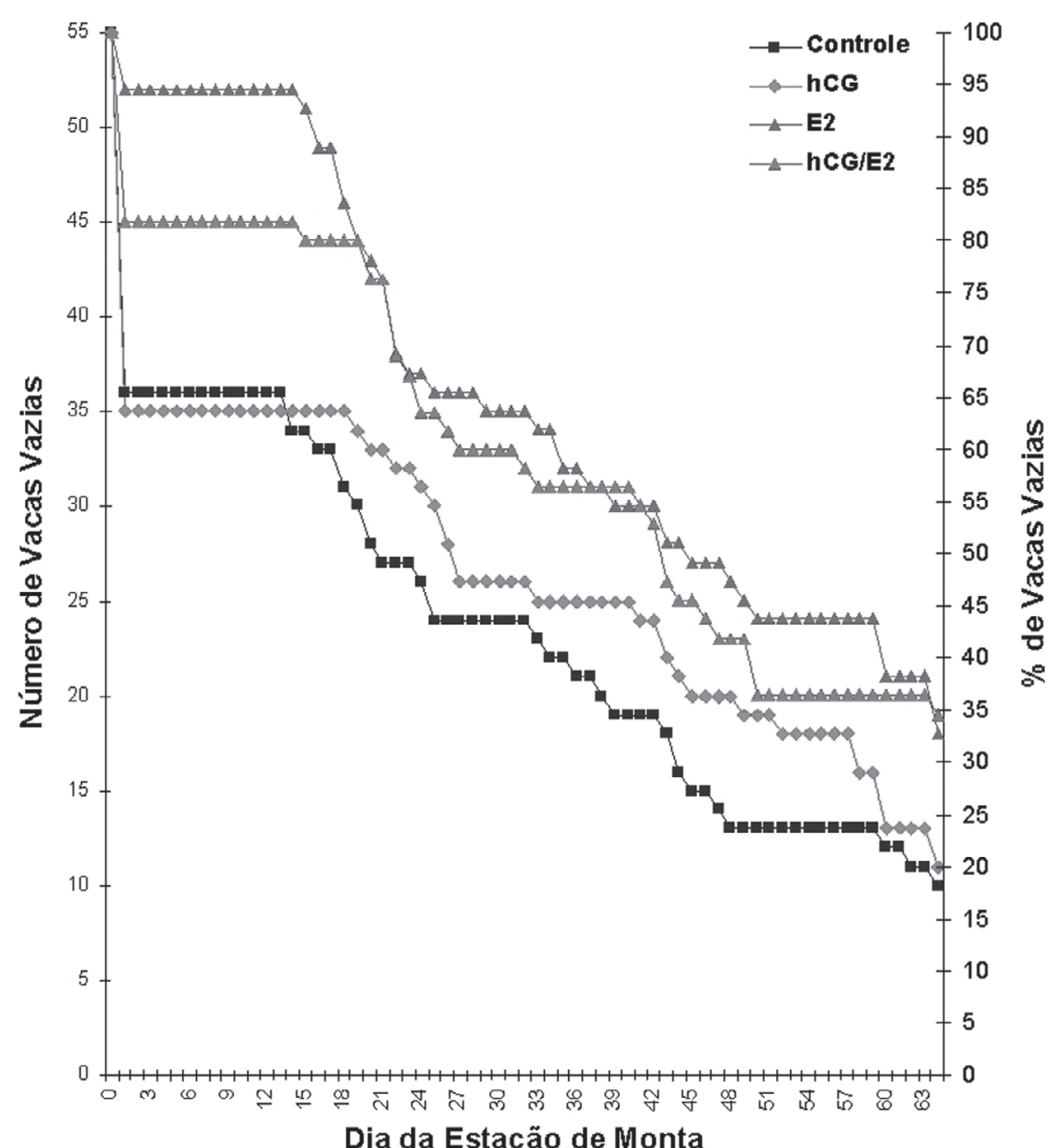

Figura 1 - Curvas de sobrevivência para as taxas de prenhez em função do dia da estação de reprodução. São Carlos, 2004

concentração de progesterona circulante. ${ }^{11,12,15}$ De fato, vacas tratadas com hCG no $\mathrm{D}_{7}$ após a IA tiveram taxa de prenhez superior à do controle e foi concluído que o efeito benéfico da hCG advém de um corpo lúteo acessório no ovário. ${ }^{11}$ Kastelic ${ }^{16}$ verificou importante aumento na taxa de prenhez em vacas suplementadas com hCG após indução da ovulação com OvSynch e IA TF Entretanto, no presente experimento a hCG não aumentou as taxas de prenhez (Tabela 2), o que permite inferir que essas taxas não foram limitadas pela concentração circulante da progesterona. Conclusão análoga foi obtida por Schmitt et al. ${ }^{12}$, que estudaram vacas submetidas a estresse térmico.

Estudos similares com fêmeas de raças européias ${ }^{12,15}$ também falharam em mostrar efeito da hCG sobre taxas de prenhez à $\mathrm{IA}_{\mathrm{TF}}$ e acumulada. Com efeito, Diaz et al. ${ }^{10}$ demonstraram não existir efeitos residuais da hCG sobre a duração do ciclo estral ou concentração de progesterona circulante nos ciclos estrais subseqüentes à sua aplicação. Portanto, e em acordo com os presentes achados (Tabela 1), não eram esperadas diferenças nas taxas de prenhez à $1^{\mathrm{a}}$ ou $2^{\mathrm{a}} \mathrm{IA}$ de repasse em resposta à um desafio com hCG após a $\mathrm{IA}_{\mathrm{TF}}$. 
Tabela 2 - Taxas de prenhez (TP) 1 à inseminação artificial (IA) em tempo fixo $\left({ }_{\text {IATF }}\right.$ ) e TP acumuladas ao final da estação $\left(\mathrm{TP}_{\text {acum }}\right)$. São Carlos, 2004

\begin{tabular}{|c|c|c|c|c|c|}
\hline \multirow{2}{*}{$\begin{array}{l}\text { Variável } \\
\text { Resposta }\end{array}$} & \multicolumn{4}{|c|}{ Fator $^{2}$} & \multirow{2}{*}{$\chi^{2}$} \\
\hline & sem hCG & com hCG & sem $E_{2}$ & $\operatorname{com} E_{2}$ & \\
\hline $\mathrm{TP}_{\mathrm{IATF}}$ & $\begin{array}{c}20,0 \% \\
(22 / 110)\end{array}$ & $\begin{array}{c}27,3 \% \\
(30 / 110)\end{array}$ & - & - & $1,61^{\mathrm{ns}}$ \\
\hline $\mathrm{TP}_{\mathrm{IATF}}$ & - & - & $\begin{array}{c}35,4 \% \\
(39 / 110)\end{array}$ & $\begin{array}{c}11,8 \% \\
(13 / 110)\end{array}$ & $15,2 *$ \\
\hline $\mathrm{TP}_{\mathrm{acum}}$ & $\begin{array}{c}74,5 \% \\
(82 / 110)\end{array}$ & $\begin{array}{c}72,7 \% \\
(80 / 110)\end{array}$ & - & - & 0,07 ns \\
\hline $\mathrm{TP}_{\mathrm{acm}}$ & - & - & $\begin{array}{c}80,9 \% \\
(89 / 110)\end{array}$ & $\begin{array}{c}66,4 \% \\
(73 / 110)\end{array}$ & $4,65^{*}$ \\
\hline
\end{tabular}
vacas dos grupos " $E_{2}$ " e "hCG/ $E_{2}$ "

Com relação ao 17 -estradiol verificou-se que ele reduziu a taxa de prenhez à $\mathrm{IA}_{\mathrm{TF}}$ e esse dano não foi revertido ao longo da estação reprodutiva (Tabela 2), pois seu efeito deletério sobre a taxa de prenhez acumulada também foi significativo $(\mathrm{P}<0,05)$. Peters ${ }^{5}$ sugeriu que a produção do 17 -estradiol folicular na fase luteínica teria influência negativa sobre o reconhecimento da prenhez. Em Bergamaschi ${ }^{17}$ o 17 . estradiol induziu a luteólise quando aplicado no $\mathrm{D}_{12}$. Caso o mesmo evento tenha ocorrido no presente experimento, isto levaria à mortalidade embrionária precoce. Fenômeno similar de perda embrionária ocorreu ao ser aplicada uma injeção de cipionato de estradiol associada à um CIDR-B aos $13 \pm 1$ dia após $\mathrm{IA}_{\mathrm{TF}}{ }^{18}$.

Interessante notar que o grupo hCG/ $\mathrm{E}_{2}$ obteve taxa de prenhez à $\mathrm{IA}_{\mathrm{TF}}(18,2 \%)$ intermediária, ou seja, superior $(\mathrm{P}<0,05)$ à do grupo $E_{2}(5,4 \%)$ e menor que às dos $G_{c}$ e $G_{h C G}$. Portanto, a despeito da potência luteolítica do $17_{\mathrm{b}}$-estradiol ${ }^{5,17}$, é cabível inferir que a ação luteotrófica da $\mathrm{hCG}^{8,11}$ proveu determinadas condições uterinas ${ }^{7}$ que favoreceram o desenvolvimento otimizado do concepto e amplificaram a produção de moléculas sinalizadoras da sua presença", prevenindo assim o desencadeamento da luteólise mesmo após indução pelo estrógeno. Corroborando esta inferência, verifica-se que nem sempre vacas Bos indicus com presença de corpo lúteo respondem aos agentes luteolíticos exógenos. Esse fato foi bem documentado com as prostaglandinas $\mathrm{F}_{2 \mathrm{a}}{ }^{19}$ e também foi verificado no presente experimento, pois três vacas mantiveram a prenhez mesmo após a aplicação do 17 -estradiol $\left(G_{\mathrm{E} 2}\right)$. Portanto, há fatores na relação concepto-unidade materna que amenizam o poder ou previnem a ação de agentes luteolíticos. Neste contexto, cabe especular sobre a importância $\mathrm{da}$ precisa cronologia dos eventos relacionados à manutenção da gestação. Assim, conceptos com desenvolvimento mais rápido efetuariam uma sinalização mais precoce, antecipando em certo grau o reconhecimento da prenhez e tornariam a vaca mais refratária aos efeitos de agentes luteolíticos.

As taxas de prenhez à $\mathrm{IA}_{\mathrm{TF}}$ após o protocolo OvSynch têm sido variáveis. Em 
bovinos de corte da raça Nelore têm sido encontradas taxas de parição entre $25 \%$ e $47,7 \%$ em função da raça do touro ${ }^{20}$; de $19,5 \%$ a $24,6 \%{ }^{21}$ e tão baixas quanto $15 \%{ }^{22}$. Cavalieri ${ }^{23}$ reportou $38,1 \%$ para rebanhos da Austrália. As principais causas de variação na resposta a esse protocolo são: a) falta de sincronização dos ciclos ovarianos entre as vacas tratadas, fruto da ocorrência de diferentes estádios de desenvolvimento folicular no momento da aplicação dos tratamentos ${ }^{24}$; b) resposta pobre ou variável em ovulação/luteinização após a $1^{\mathrm{a}}$ injeção de $\mathrm{GnRH}^{25}$; c) resposta pobre ou variável das vacas Nelore à ação luteolítica da $\mathrm{PGF}_{2 \mathrm{a}}$ exógena ${ }^{19}$; d) Ocorrência de luteólise expontânea entre a $1^{a}$ e $2^{a}$ doses de $G n R H^{26}$ e e) função ovariana anormal causada por balanço energético negativo ${ }^{7}$. Foi enfatizado a importância dos fatores relativos à indução da ovulação pelo $\mathrm{GnRH}$, de modo que subdosagens ou baixa potência de seus análogos poderiam causar falhas na resposta devidas a: incapacidade do GnRH induzir um pico de LH com magnitude suficiente para causar a ovulação; incapacidade do folículo em responder com ovulação à esse pico de LH; indução precoce da ovulação com a liberação de um ovócito ainda imaturo e formação de um corpo lúteo com função esteroidogênica sub-normal ${ }^{23}$.

No presente experimento, as taxas de prenhez à IA $\mathrm{TF}_{\mathrm{TF}}$ observadas para os grupos controle (OvSynch) e hCG (OvSynch + hCG) estiveram nos limites superiores dos intervalos mais freqüentemente reportados para bovinos de corte. Entretanto, Baruselli et al. ${ }^{22}$ reportaram taxas de prenhez superiores aos $60 \%$ após $\mathrm{IA}_{\mathrm{TF}}$ com o uso de protocolos de indução da ovulação baseados em progestágenos e levantaram dúvidas sobre a aplicabilidade do OvSynch para bovinos de corte em regiões tropicais, especialmente em rebanhos com alta proporção de vacas em anestro ou de novilhas, o que corroborou relato prévio ${ }^{27}$.

Ao final da estação reprodutiva de 64 dias as taxas de prenhez dos grupos controle e hCG foram equivalentes àquela $(79,8 \%)$ obtida para vacas de corte na
Austrália após uma estação reprodutiva com 56 dias de duração ${ }^{23}$ e foram superiores aos $59,7 \%$ encontrados para vacas Nelore após uma estação de monta com 60 dias. ${ }^{21}$ Nesse último estudo aumentou-se a taxa de prenhez para $80,1 \%$ por meio da remoção temporária do bezerro quando da $\mathrm{IA}_{\mathrm{TF}}$ que iniciou a estação de monta. ${ }^{21}$

Outra limitação potencial do protocolo OvSynch é a ocorrência subsequente de ciclos estrais de curta duração (menores de 18 dias) devido a função luteínica subnormal ${ }^{28}$, acarretando alto percentual $(31,8 \% \text { a } 51 \%)^{23,28}$ de inseminações de repasse antes dos 18 dias após a $\mathrm{IA}_{\mathrm{TF}}$. No presente experimento, ocorreram $10,7 \%$ de ciclos curtos, permitindo inferir que a dose e potência do análogo do GnRH (8 $\mathrm{mg}$ do acetato de buserelina) adotadas foram adequados.

As taxas de concepção às IA de repasse (Tabela 1) foram mais altas $(\mathrm{P}<0,05)$ do que à $\mathrm{IA}_{\mathrm{TF}}$. No caso de uma estação reprodutiva relativamente curta (64 dias), o impacto da baixa concepção inicial pode limitar a obtenção de uma taxa de prenhez otimizada ao final da estação. Ressalta-se que além dos fatores ligados ao protocolo de indução da ovulação previamente discutidos, especula-se a influência negativa de fatores estressores sobre a concepção. Foram destacados os efeitos climáticos (altas temperaturas, umidade, etc.) e de manejo (movimentação, contenção) sobre o comportamento da monta, do estro e da ovulação ${ }^{1}$, pois esses agentes estressores alteram a freqüência e a amplitude dos pulsos de LH e de liberação de GnRH retardando o pico pré-ovulatório do LH com subsequente redução na expressão do estro e da ovulação. Este mecanismo explicaria as baixas taxas de serviço e de fertilidade dos programas de IA aplicados em bovinos Bos indicus ${ }^{29}$. No presente experimento várias situações adicionais de estresse foram aplicadas, tais como: intensa movimentação e contenção das fêmeas para a avaliação ginecológica, seleção, indução da ovulação, $\mathrm{IA}_{\mathrm{TF}}$, mudança de piquete e apartação para as IAs de repasse. Foi documentado, em 
vacas leiteiras ${ }^{30}$, que esse tipo de estresse ("da experimentação") reduz sobremaneira as taxas de fertilização e de prenhez. Além disso, após a $\mathrm{IA}_{\mathrm{TF}}$ houve um re-arranjo para a formação dos quatro grupos (lotes) experimentais, o que modificou a estrutura social hierárquica do rebanho e pode ter conseqüências negativas no desempenho reprodutivo das vacas. ${ }^{31}$ Portanto, é cabível inferir algum efeito do "estresse" sobre as taxas de prenhez obtidas neste experimento, embora as taxas do Gc e $G_{h C G}$ foram comparáveis aos valores reportados por outros investigadores. ${ }^{21,23}$

\section{Conclusões}

Há a necessidade do desenvolvimento de estratégias para a redução da mortalidade embrionária precoce em bovinos, pois os protocolos testados neste experimento não aumentaram as taxas de concepção ou de prenhez. Assim, a otimização da função luteínica de vacas Nelore sem bezerro ao pé, em boa condição corporal e alta taxa de ciclicidade pela hCG não foi efetiva demonstrando que as concentrações circulantes de progesterona não limitaram as taxas de prenhez. Por sua vez, a reprogramação ovariana mediada pelo $17_{\mathrm{b}}$ estradiol após uma $\mathrm{IA}_{\mathrm{TF}}$ exige cautela, pois sua aplicação relacionou-se às menores taxas de prenhez e, neste caso a suplementação gonadotrófica pela hCG minimizou esses efeitos deletérios.

\section{Agradecimentos}

À EMBRAPA PECUÁRIA SUDESTE pela cessão dos animais e fornecimento da maioria dos fármacos usados, bem como ao Centro Paulista de Desenvolvimento Farmacotécnico, que doou o 17-b estradiol;

À FAPESP pelo apoio financeiro (Proc.99/03313-9 e Proc. 02/ 08363-0).

\section{Service, conception and pregnancy rates of nelore cows treated with human corionic gonadotropin and estradiol-17 $\beta$ after timed artificial insemination}

\begin{abstract}
Strategies to reduce embryo mortality in cattle have been evaluated. Nelore (Bos taurus indicus) cows were submitted to timed artificial insemination (TAI $-\mathrm{D}_{0}$ ) after a $\mathrm{GnRH} / \mathrm{PGF}_{2 \mathrm{a}} / \mathrm{GnRH}$ protocol. Cows were divided in the following groups: Control (Gc; $n=55)$, hCG $\left(\mathrm{G}_{\mathrm{hCG}} ; \mathrm{n}=55\right)$ received $3000 \mathrm{IU}$ of $\mathrm{hCG}$ on $\mathrm{D}_{5}, \mathrm{E}_{2}\left(\mathrm{G}_{\mathrm{E} 2} ; \mathrm{n}=55\right)$ received $5 \mathrm{mg}$ of Estradiol- $17_{\mathrm{b}}\left(\mathrm{E}_{2}\right)$ on $\mathrm{D}_{12}$ and hCG $/ \mathrm{E}_{2}\left(\mathrm{G}_{\mathrm{hCG} / \mathrm{E} 2}\right.$; $\mathrm{n}=55)$ received $\mathrm{hCG}$ on $\mathrm{D}_{5}$ and $\mathrm{E}_{2}$ on $\mathrm{D}_{12}$. TAI represented the $1^{\text {st }}$ day of a 64 day breeding season. The $\chi^{2}$ test was applied to compare mating rate (MR), conception rate (CR) and pregnancy rate (PR) to TAI and AIs after natural estrus within the season as well as their accumulated figures at the end of the season. MR and CR for AIs within season did not differ $(\mathrm{P}>.10)$. PR to TAI differed $(\mathrm{P}<.05)$ among groups. $G_{\mathrm{F} 2}$ showed the lowest rate $(5,4 \%)$. Gc $(34.5 \%)$ and $\mathrm{G}_{\mathrm{hCG}}(36.5 \%)$ tended $(P<.10)$ to be higher than $\mathrm{G}_{\mathrm{hCG} / \mathrm{E} 2}(18.2 \%)$. There was no effect of hCG on pregnancy rates. $\mathrm{E}_{2}$ reduced PR to TAI and at the end of season which means that cows receiving $\mathrm{E}_{2}$ $\left(\mathrm{G}_{\mathrm{E} 2}+\mathrm{G}_{\mathrm{hCG} / \mathrm{E} 2}\right)$ had lower $(66.4 \%$; $<<.05)$ PR than cows not receiving $\mathrm{E}_{2}\left(\mathrm{G}_{\mathrm{C}}+\mathrm{G}_{\mathrm{hCG}} ; 80.9 \%\right)$. In conlusion, the strategies to manipulate luteal and follicular function herein tested did not increase pregnancy rates in Nelore cows submitted to a TAI.
\end{abstract}

Key words:

Cattle.

Embryo mortality.

Estradiol.

Chorionic gonadotropin. 


\section{Referências}

1 BÓ, G. A.; BARUSELLI, P. S.; MARTINEZ, M. F. Pattern and manipulation of follicular development in bos indicus cattle. Animal Reproduction Science, v. 78, p. 307-326, 2003.

2 ANUALPEC. Anuário da Pecuária Brasileira - FNP. São Paulo: FNP Consultoria \& Comércio, 1994-2004.

3 DUNNE, L. D.; DISKIN, M. G.; SREENAN, J. M. Embryo and foetal loss in beef heifers between day 14 of gestation and full term. Animal Reproduction Science, v. 58, n. 1, p. 39-44. 2000.

4 BINELLI, M. et al. Antiluteolytic strategies to improve fertility in cattle. Theriogenology, v. 56, p. 1451-1463, 2001.

5 PETERS, A. R. Embryo mortality in the cow. Animal Breeding Abstracts, v. 64, p. 587-598. 1996.

$6 \mathrm{MACHADO}$, R. et al. Strategies to prevent the presence of a dominant follicle between days 15 and 20 of the cow estrous cycle. In: INTERNATIONAL CONGRESS ON ANIMAL REPRODUCTION, 15., 2004, Porto Seguro. Anais... Belo Horizonte: Colégio Brasileiro de Reprodução Animal, 2004. p. 118.

7 MANN, G. E.; LAMMING, G. E. The influence of progesterone during early pregnancy in cattle. Reproduction of Domestic Animals, v. 34, p. 269274, 1999.

8 MANN, G. E.; LAMMING, G. E.; FISCHER, P. A. Progesterone control of interferon-t production during early pregnancy in the cow. Journal of Reproduction and Fertility, Abstract series 21, 1998. Abstract 37.

9 GEISERT, R. D. et al. Characterization of the uterine environment during early conceptus expansion in the bovine. Animal Reproduction Science, v. 16, n. 1, p. 11-25. 1988.

10 DIAZ, T. et al. HCG-induced alterations in ovarian folicular dynamics during the estrous cycle of heifers. Journal of Animal Science, v. 76, n. 7, p. 1929-1936, 1998.

11 RAJAMAHENDRAN, R.; SIANANGAMA, P. C. Effect human Corionic Gonadotrophin on dominant follicles in cows. Formation of accessory corpora lutea, progesterone production and pregnancy rates. Journal of Reproduction and Fertility, v. 95, n. 2, p. 577-584, 1992.

12 SCHMITT, E. J. P. et al. Diferential response of the luteal phase and fertility in cattle following ovulation of the first wave follicle with hCG or an agionist of $\mathrm{GnRH}$. Journal of Animal Science, v. 74, n. 5, p. 1074-1083, 1996.

13 PURSLEY, J. R.; MEE, M. O.; WILTBANK, M. C. Synchronization of ovulation in dairy cows using PG F2a and GnRH. Theriogenology, v. 44, p. 915-923. 1995.
14 SAS INSTITUTE. SAS/STAT: user's guide. Version 6. 4. ed. Cary, 1993. v. 2, p. 891-1686.

15 BREUEL, K. F.; SPITZER, J. C.; HENRICKS, D. M. Systemic progesterone concentration following hcg administration at various times during the estrous cycle in beef heifers. Journal of Animal Science, v. 67, n. 6 , p. 1564-1572, 1989.

16 KASTELIC, J. P. Folliculogenesis in cattle. In: SIMPÓSIO INTERNACIONAL DE REPRODUÇÃO ANIMAL APLICADA, 1., 2004, Londrina. Anais... São Paulo: Faculdade de Medicina Veterinária e Zootecnia, Universidade de São Paulo, 2004. p. 17-25.

17 BERGAMASCHI, M. A. C. M. Estratégias hormonais para otimizar a função luteínica de vacas da raça Nelore após a sincronização do estro. 2005. 111 f. Tese (Doutorado) - Faculdade de Ciências Agrárias e Veterinárias, Universidade Estadual Paulista, Jaboticabal, 2005.

18 COLAZO, M. G. et al. Follicle dynamics, estrus and ovulation in CIDR-treated cows given ECP, GnRH, or progesterone during diestrus. In: INTERNATIONAL CONGRESS ON ANIMAL REPRODUCTION, 15. 2004, Porto Seguro. Anais... Belo Horizonte: Colégio Brasileiro de Reprodução Animal, 2004. p. 330.

19 PINHEIRO, O. L. et al. Estrous behaviour and the estrus-to- ovulation interval in nelore cattle (bos indicus) with natural estrus or estrus induced with prostaglandin F2a or norgestomet and estradiol valerate. Theriogenology, v. 49, p. 667-681, 1998.

20 FERNANDES, P. et al. Timed artificial insemination in Nelore cows. Arquivos da Faculdade de Veterinária da UFRGS, v. 25, n. 1, p. 223, 1997.

21 VILELA, E. R. et al. Effect of progesterone device on conception at timed $\mathrm{Al}$ protocol and pregnancy rate during breeding season in sucled Nelore cows. In: INTERNATIONAL CONGRESS ON ANIMAL REPRODUCTION, 15., 2004, Porto Seguro. Anais... Belo Horizonte: Colégio Brasileiro de Reprodução Animal, 2004. p. 368.

22 BARUSELLI, P. S. et al. Inseminação artificial em tempo fixo em bovinos de corte. . In: SIMPÓSIO INTERNACIONAL DE REPRODUÇÃO ANIMAL APLICADA, 1., 2004, Londrina. Anais... São Paulo: Faculdade de Medicina Veterinária e Zootecnia, Universidade de São Paulo, 2004. p. 155-165.

23 CAVALIERI, J. Induction of ovulation in Bos indicus and Bos taurus cattle following synchronization of oestrous cycles with emphasis on Australian studies. In: SIMPÓSIO INTERNACIONAL DE REPRODUÇÃO ANIMAL APLICADA, 1., 2004, Londrina. Anais... São Paulo: Faculdade de Medicina Veterinária e Zootecnia, Universidade de São Paulo, 2004. p. 82-104.

24 BÓ, G. A. et al. Exogenous control of follicular wave emergency in cattle. Theriogenology, v. 43, p. 3140, 1995.

25 BÓ, G. A. et al. The control of follicular wave 
development for self-appointed embryo transfer programs in cattle. Theriogenology, v. 57, p. 53-72, 2002.

26 MARTINEZ, M. F.; BÓ, G.; MAPLETOFT, R. J. Synchronization of follicular wave emergence and ovulation for reproductive biotechnology. . In: SIMPÓSIO INTERNACIONAL DE REPRODUÇÃO ANIMAL APLICADA, 1., 2004, Londrina. Anais... São Paulo: Faculdade de Medicina Veterinária e Zootecnia, Universidade de São Paulo, 2004. p. 26-55.

27 BARROS, C. M.; MOREIRA, M. B. P.; FERNANDES, P. Pharmacological manipulation of estrous cycle to improve artificial insemination or embryo transfer programs. Arquivos da Faculdade de Veterinária da UFRGS, v. 26, n. 1, p. 179-198, 1998. Suplemento.

28 MACMILLAN, K. L.; SEGWAGWE, B. V. E.; PINO, C. S. Associations between the manipulation of patterns of follicular development and fertility in cattle. Animal Reproduction Science, v. 78, p. 327-344, 2003.

29 GALINA, C. S.; ORIHUELA, A.; RUBIO, I. Behavioural trends affecting oestrus detection in zebu cattle. Animal Reproduction Science, v. 42, p. 465470, 1996.

30 MANN, G. E. Pregnancy rates during experimentation in dairy cows. The Veterinary Journal, v. 161, p. 301305, 2001.

31 DOBSON, H.; SMITH, R. F. Stress and subfertility. Reproduction in Domestic Animals, v. 33, p. 107111, 1998. 